\title{
An investigation of the location of fatigue initiation - deterministic and probabilistic aspects
}

\author{
Kristoffer Karlén and Mårten Olsson
}

\author{
Department of Solid Mechanics \\ Royal Institute of Technology (KTH) \\ Stockholm, Sweden
}

\begin{abstract}
Fatigue limit tests where there are two competing fatigue failure locations, two notches of different size, are performed in this paper. The normal distribution is used for describing the experimental outcome, where the largest occurring stress value or the gradient adjusted stress is used as a stress measure. Another model for describing the experimental outcome is the Weakest Link (WL)-integral where the integration is performed over the specimen surface area or the specimen volume. The longitudinal positions of the estimated fatigue failure sites are measured and investigated as well.

Neither the normal distribution (point stress and gradient adjusted point stress), nor the WL-integral can explain the experimental outcome from the competing notch tests. They all predict the same trend, but not the same trend as in the experiments. When looking at the estimated longitudinal location of fatigue failure it can be seen that it differs considerably from experiment to experiment. If the stress is evaluated at the estimated fatigue failure location, it is seen that the local stress is much lower than the maximal stress. The load cases where there should be an equal amount of failures in both notches according to the different models do not agree with the experimental findings. It is concluded that stress based continuum models can not describe the experimental outcome when there are competing fatigue failure sites (notches) present.
\end{abstract}




\section{Introduction}

Scatter is always present in High Cycle Fatigue (HCF), both for finite life [1, 2] and for the fatigue limit regime [3-6]. This scatter is quantified using experiments $[1,3,5,6]$. The experimental scatter in the fatigue limit regime is then modeled using for instance the Weakest Link (WL)-integral, [7, 8]. The finite life scatter is usually modeled using a lognormal distribution. Scatter for finite life, where the load is constant and the life is varying is different from the fatigue limit regime where an experiment is treated as either a failure or a run-out. Note that the fatigue limit scatter refers to the scatter in load, i.e. depending on the stress levels all, some or none specimen fail.

In this paper, the experimental scatter is to be analyzed in another way. Focus is set at the scatter in fatigue failure location. Once the failure locations are estimated the stress can be evaluated and the local fatigue failure probability can be computed. The scatter in the fatigue failure locations can be expressed as scatter in local stresses and hence fatigue failure probabilities.

A specimen with two competing fatigue failure sites, two notches of different size, is used here. This specimen has been used in $[1,3,4,9]$ for determining both the fatigue limits (one for each notch) and the finite life behavior. A set of experiments where fatigue failure could occur in both notches were performed.

The normal distribution is used as a fatigue limit model when using the largest occurring point stress value $[1,4,10]$. This gives a rather conservative prediction. If the largest point stress is reduced with its stress gradient, a better prediction is obtained, $[1,4]$. Another way to model the fatigue limit scatter is to use the WL-integral, $[7,8]$. The integration can be performed over the specimen surface area $[1,4,9,11,12]$ or as a volumetric entity $[1,3,4,9,13,14]$.

In order to better understand the experimental scatter in initiation position, it is analyzed in conjunction with stresses and probability of failure.

The goals of this paper can be summarized as: $i$ ) investigating the scatter in fatigue failure locations, $i$ i) making a qualitative comparison between different fatigue limit models when there are two fatigue failure locations, two notches in this case, present and $i i i$ ) increase the understanding of scatter in the fatigue initiation location.

The first section of this paper is the experimental investigation where both the fatigue limit tests and the experiments where failure can occur in both notches are presented. After this, the fatigue failure initiation locations are presented. This is followed by simulations and a presentation of the fatigue limit models used in this paper. Next, the failure location analysis is presented where gradient parameters are investigated. This is followed by a section dealing with the fatigue lives for the broken specimens. The last section contains the discussion, summary and conclusions. 


\section{Experimental investigation}

The material used is Uddeholm IMPAX supreme, corresponding to AISI P20 mod, with chemical composition as in Table 1. The low sulphur content, high purity and good homogeneity gives this steel excellent fatigue endurance. The yield strength, $R_{p 0.2}$, is $850 \mathrm{MPa}$, the ultimate tensile strength, $R_{m}$, is 1000 $\mathrm{MPa}$ and Young's modulus, $E$, is $205 \mathrm{GPa}$. This steel is used as moulds for plastics for example.

Table 1: Chemical composition of IMPAX supreme, given in wt\%.

\begin{tabular}{ccccccc}
\hline $\mathrm{C}$ & $\mathrm{Si}$ & $\mathrm{Mn}$ & $\mathrm{Cr}$ & $\mathrm{Ni}$ & $\mathrm{Mo}$ & $\mathrm{S}$ \\
\hline \hline 0.37 & 0.30 & 1.40 & 2.0 & 1.0 & 0.20 & 0.008 \\
\hline
\end{tabular}

The specimen used, see Fig. 1, is tailored for the fatigue limit tests. This specimen has been designed for investigating the volume effect, which is why the notches have different size (different diameters). The axi-symmetric specimen is inserted into a rotating bending machine in order to conduct the fatigue tests. The specimen is manufactured using turning from as-received rods. The elastic stress concentration factor for bending, $K_{t}$, is 1.48 for notch $A$ and 1.32 for notch $B$, according to [15].

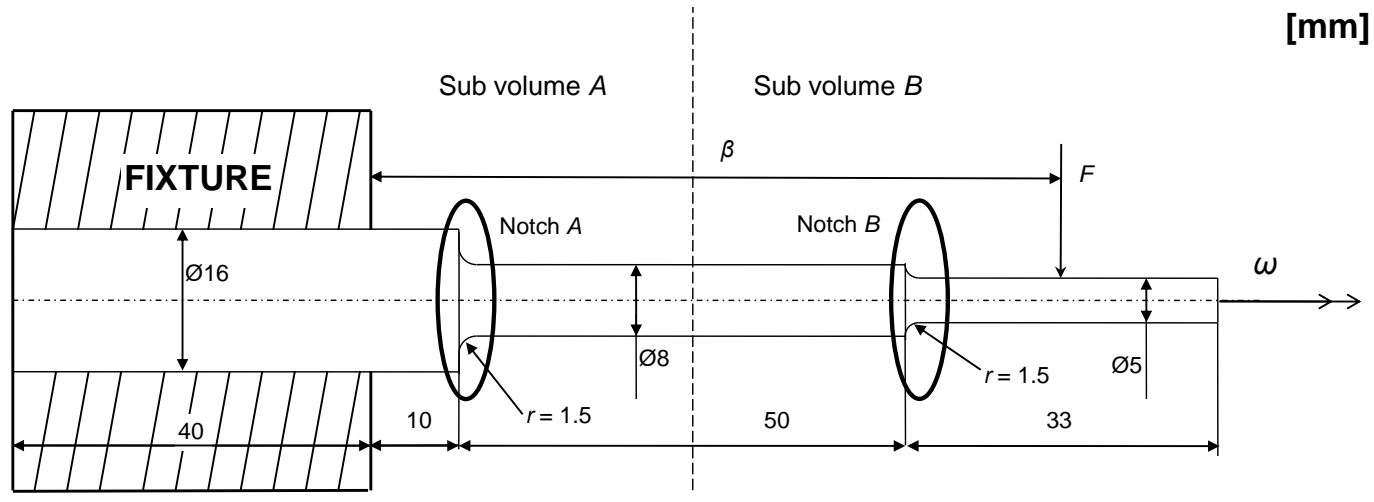

Figure 1: Schematic of the specimen used in the experiments.

By changing the location of the load, $\beta$, the fatigue failure location can be changed. If $\beta$ is small, failure will occur in notch $A$ and if $\beta$ is large the failure will occur in notch $B$.

\subsection{Fatigue limit tests}

Fatigue limit tests, FL-tests, presented in [3] have been analyzed in this paper. The experiments have been performed according to the staircase method, [16], 
and the experimental outcome is presented in Fig. 2. A run-out is defined as specimen that did not break after $2 \cdot 10^{6}$ cycles and the failure criterion used is complete failure. The rotational speed in the fatigue tests was 3,000 rpm corresponding to a frequency of $50 \mathrm{~Hz}$.
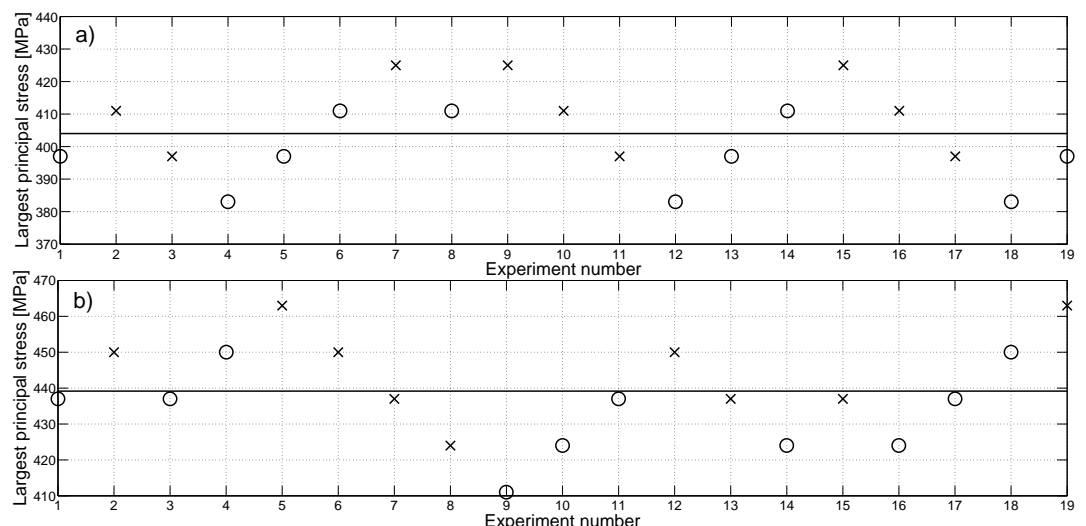

Figure 2: Experimental outcome from the staircase tests performed, ring indicates a run-out and a cross $(x)$ failure and a) is for notch $A$ and $b$ ) is for notch $B$.

Using equations presented in [16] the mean and standard deviation for the fatigue limit in notch $A$ were $404 \mathrm{MPa}$ and $15.8 \mathrm{MPa}$, respectively. For notch $B$ they were $439 \mathrm{MPa}$ and $17.8 \mathrm{MPa}$. The experimental outcome presented in Fig. 2 is summarized in Table 2 .

Table 2: Outcome from the fatigue limit tests.

\begin{tabular}{lccccccccc}
\hline & \multicolumn{3}{c}{ Notch $A(\beta=72$} & $\mathrm{mm})$ & \multicolumn{4}{c}{ Notch $B(\beta=85 \mathrm{~mm})$} \\
Stress level $[\mathrm{MPa}]$ & 383 & 397 & 411 & 425 & 411 & 424 & 437 & 450 & 463 \\
\hline \hline Failures & 0 & 3 & 3 & 3 & 0 & 1 & 3 & 3 & 2 \\
Run-outs & 3 & 4 & 3 & 0 & 1 & 4 & 4 & 2 & 0 \\
\hline
\end{tabular}

\subsection{Experiments with fatigue failure in both notches}

In order to analyze the predictive capabilities of the different fatigue limit models, experiments where fatigue failure could occur in both notches were performed. These tests are called "competing notch tests", CN-tests. The load cases were chosen so that there would be both run-outs and failures for the different load cases. The load cases presented in Table 3 have been investigated where load case 7 is taken from [3].

In Table 3 it can be seen that most specimens fail in notch $B, 33$ out of 42 failures. For higher values on $\beta$, most fatigue failures occur in notch $B$ but some 
Table 3: Outcome from the competing notch tests.

\begin{tabular}{lccccccc}
\hline Load case & 1 & 2 & 3 & 4 & 5 & 6 & 7 \\
\hline \hline$\beta[\mathrm{mm}]$ & 78 & 79 & 80 & 81 & 82 & 83 & 84 \\
$F[\mathrm{~N}]$ & 206 & 200 & 197 & 195 & 196 & 186 & 180 \\
Failure in $A$ & 3 & 3 & 1 & 1 & 0 & 1 & 0 \\
Failure in $B$ & 0 & 4 & 7 & 5 & 3 & 4 & 10 \\
Run-outs & 4 & 3 & 2 & 4 & 7 & 5 & 3 \\
\hline
\end{tabular}

occur in notch $A$. There is a transition point where all failures occur in notch $A$, load case 1 .

\section{Failure location measurements}

The longitudinal position of the estimated fatigue initiation location was measured. The definition of the initiation location, shown in Fig. 3a, is taken from [17]. It is assumed that the initiation location is on the edge of fracture surface of the specimen, the red line in Fig. 3b.
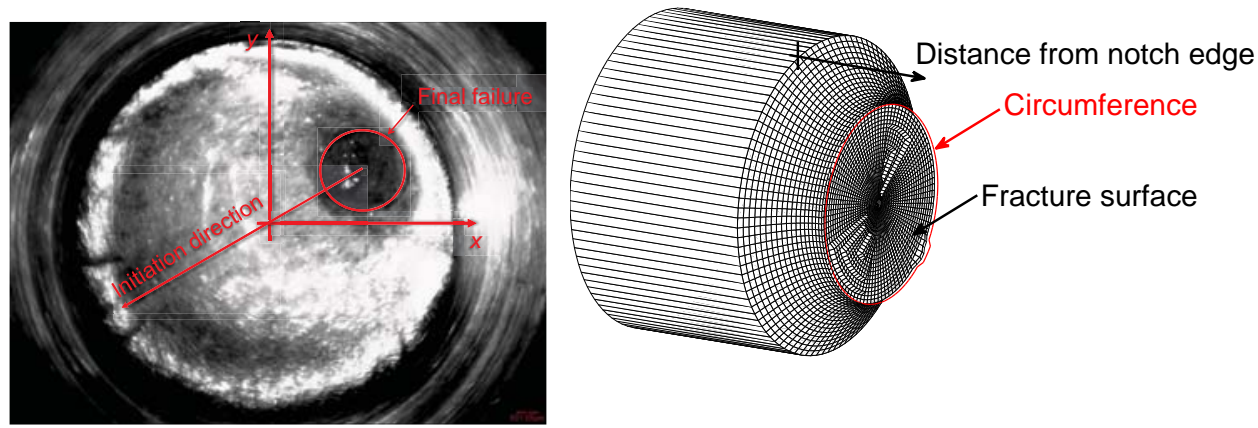

(a) Fatigue initation location.

(b) Perspective view of the failure locations presented in Fig. 4 and 5.

Figure 3: Definition of the fracture surface measurements.

In order to perform the measurements, the specimens were mounted in a fixture and the initiation location was measured as distance from the notch edge, see Fig. 3b. A dial indicator, with a resolution of $\pm 0.01 \mathrm{~mm}$, was used for the measurements. For each fracture surface the estimated initiation location, largest and smallest distance on the edge and the largest and smallest distance over the entire fracture surface were measured, which is shown in Fig. 3b. In Fig. 4 the initiation location are presented for both notches.

In Fig. 4 it can be seen that the fatigue failures for the different notches initiates on the same interval. The notch geometry (i.e. height and radius) is the same for both notches. Fig. 5 shows the estimated initiation location for 

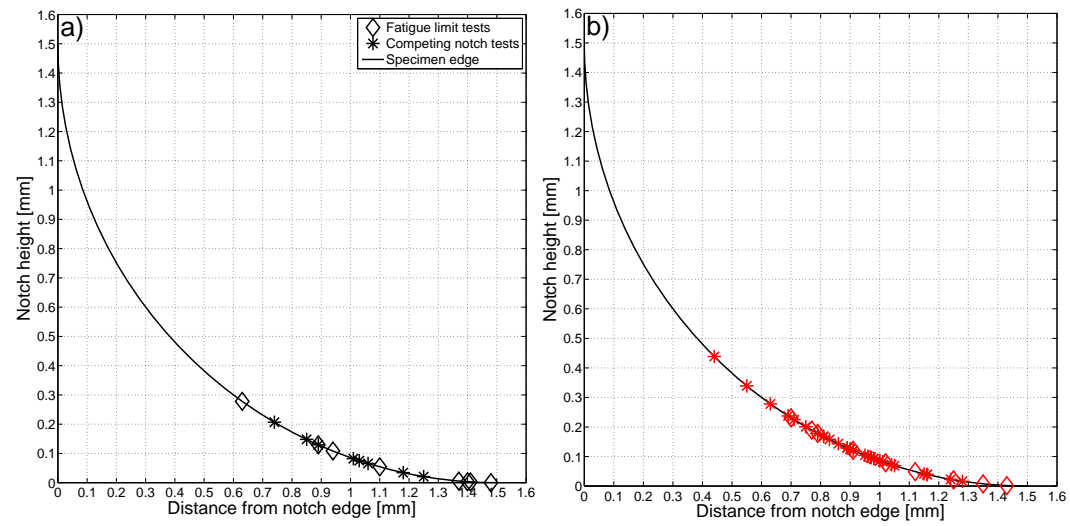

Figure 4: Longitudinal fatigue initiation location in the notch, a) is notch $A$ and b) is notch $B$.

both notches after the tests have been finalized. The results are presented using the failure probabilities of the respective test series (with the same loading).

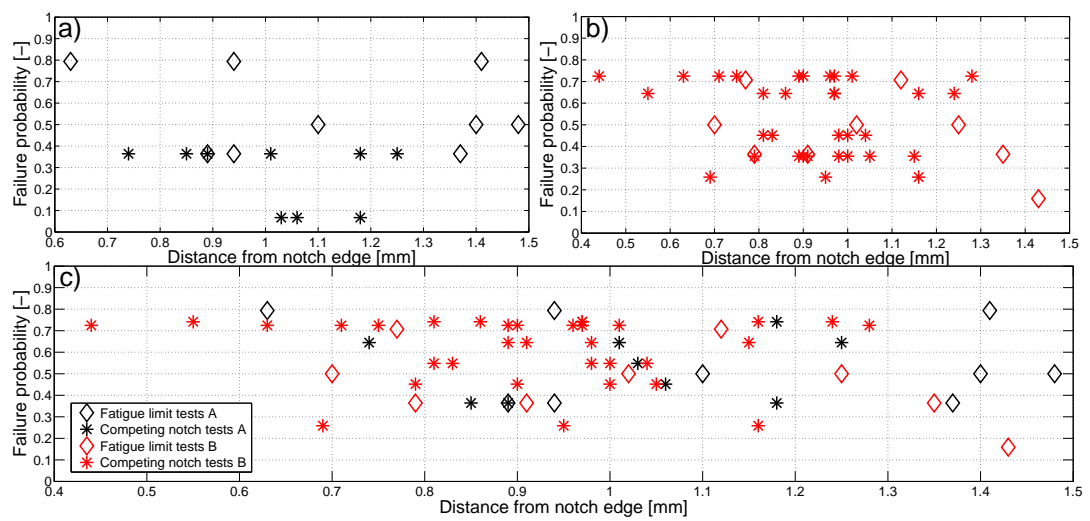

Figure 5: Fatigue failure location measurements. The failure probability corresponds to the failure probability from the experiments and a) is for notch $A, \mathrm{~b}$ ) is for notch $B$ and c) is for notches $A$ and $B$ pooled together.

In Fig. 5 it can be seen that the failure initiation locations are in the same range for both the fatigue limit tests and the competing notch tests. In Fig. 6 the estimated fatigue failure locations along with maximal and minimal values for the global and the edge measurements for the fatigue limit tests are presented.

A substantial amount of scatter is present in Fig. 6. The measurements presented in this figure can be seen as confidence bounds. The same entities for the competing notch tests are shown in Fig. 7.

In Fig. 6 and 7 it can be seen that the failure locations are different from 

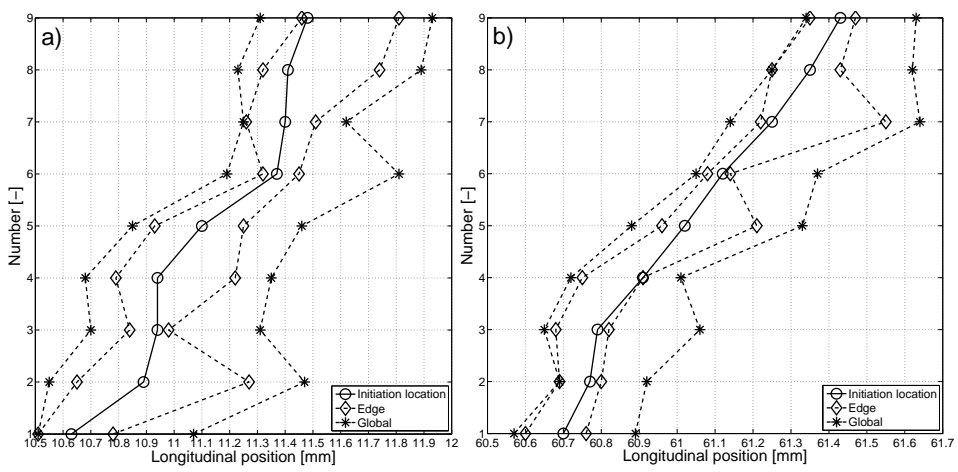

Figure 6: Sorted fatigue failure location measurements from the fatigue limit tests. Edge indicates measures on the the edge and global are the largest occurring values for each failure location measurement and, finally, a) is for notch $A$ and $\mathrm{b}$ ) is for notch $B$.
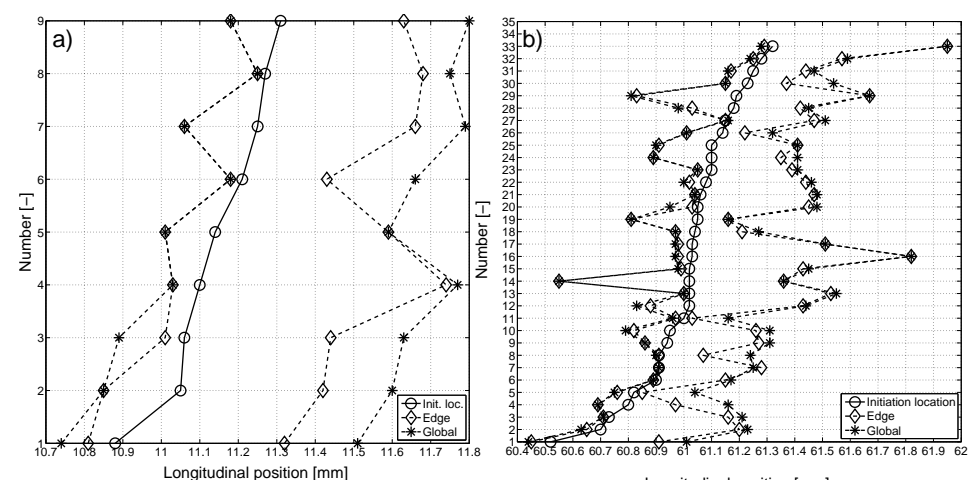

Figure 7: Sorted fatigue failure site measurements from the competing notch tests. Edge indicated measures on the edge and global are the largest occurring values for each failure site measure. a) is for notch $A$ and b) is for notch $B$. The numbers on the $y$-axis indicate number of failed specimens, which is 9 for notch $A$ and 33 for notch $B$.

experiment to another.

\section{Simulations}

The experiments were analyzed using the FE-software ABAQUS and the results were post-processed using Matlab. The element types used were C3D8 and C3D6, [18], which are eight noded brick elements and wedge elements, respectively. The wedge elements were used in the middle of the specimen in order to simplify the meshing. The mesh was checked for convergence. 


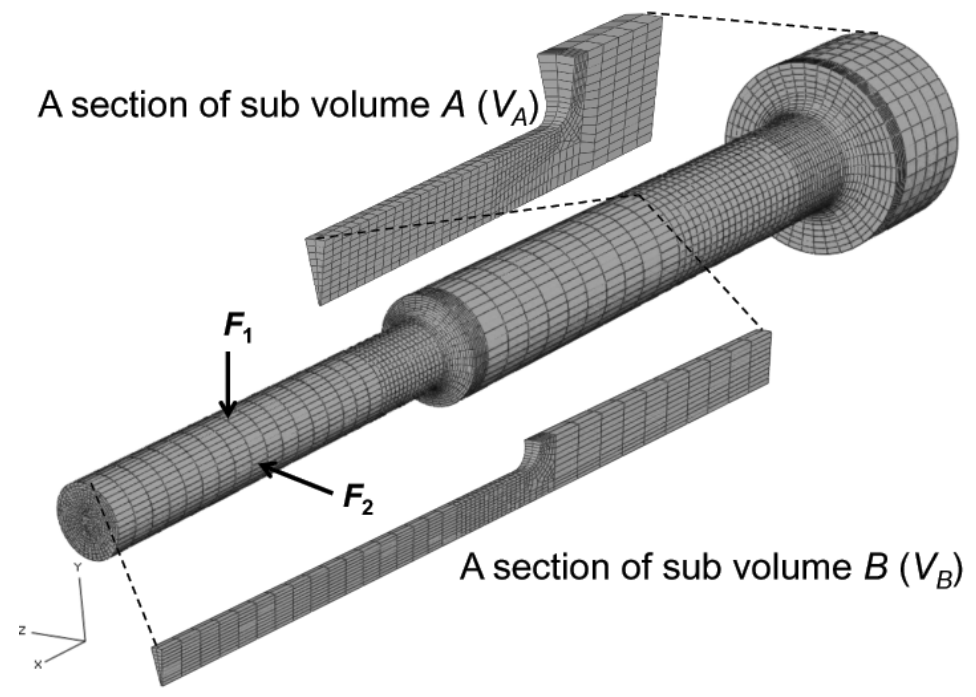

Figure 8: FE-model of the specimen with the element sets corresponding to the notch sub volumes indicated.

As can be seen in Fig. 8, the mesh was divided into two different sub meshes $(A$ and $B)$ in accordance with the sub volumes of Fig. 1. The different sub volumes, $A$ and $B$, were used for evaluation of local contributions to the total failure probability $p_{f, \text { tot }}$, i.e. $p_{f, A}$ and $p_{f, B}$. This can be expressed as

$$
p_{f, \text { tot }}=1-\left(1-p_{f, A}\right)\left(1-p_{f, B}\right) .
$$

In order to determine the failure probability for a sub-volume and, say $A$, the slice-shaped section in Fig. 8 can be used. The following relation was used

$$
p_{f, A}=1-\left(1-p_{f, A \text { section }}\right)^{N} .
$$

In Eq. (2), $p_{f, A}$ indicates the failure probability for the whole sub-volume and $p_{f, A s e c t i o n}$ the failure probability for a section and $N$ is the total number of sections, which here is 20. The total failure probability is then given in Eq. (1).

The stress tensor history due to a dead load on a rotating specimen can be found as the result of a combination of the stresses from two load cases [19], with equal perpendicular forces, $F_{1}$ and $F_{2}$, where $F_{1}=F_{2}$. The resulting stress field from one loading is the $90^{\circ}$-rotation of the other. The total stress history is

$$
\boldsymbol{\sigma}(\mathbf{x}, \tau)=\boldsymbol{\sigma}_{1}(\mathbf{x}) \cos (\tau)+\boldsymbol{\sigma}_{2}(\mathbf{x}) \sin (\tau),
$$

where $\boldsymbol{\sigma}$ denotes the stress tensor at an arbitrary point $\mathbf{x}$ at pseudo time $\tau$, $\sigma_{1}(\mathbf{x})$ and $\boldsymbol{\sigma}_{2}(\mathbf{x})$ denote the stress solutions due to loading 1 and 2 , respectively. 
For the current stress history, with "alternating" bending type stresses, the following expression is used for determination of the effective stress

$$
\sigma_{\mathrm{eff}, \Delta \sigma_{1}}=\max _{t}\left(\sigma_{1}\right) \leq \sigma_{\mathrm{eff}, \Delta \sigma_{1}}^{\mathrm{crit}}
$$

In Eq. (4) $\sigma_{1}$ indicates the largest principal stress. The criterion in Eq. (4) is only applicable to alternating stress histories. The largest principal stress criterion is used here, because the choice of effective stress does not have a large influence at the fatigue limit, see $[3,10]$.

\section{The fatigue limit models}

In order to model the fatigue limit scatter, the WL-integral can be used. The failure probability using an area approach can be expressed as

$$
p_{f, \text { surface }}=1-\exp \left(-\int_{A_{\text {surf }}}\left(\frac{\sigma_{\text {eff }}-\sigma_{\mathrm{th}, \text { surf }}}{\sigma_{\mathrm{u}, \text { surf }}}\right)^{n} \frac{\mathrm{d} A}{A_{\text {ref }}}\right),
$$

where $\sigma_{\text {eff }}$ is an effective stress, $\sigma_{\text {th,surf }}$ is a threshold stress, $\sigma_{\mathrm{u}, \text { surf }}$ is a location parameter, $n$ is material parameter controlling the scatter and $A_{\text {ref }}$ is an arbitrary reference area. The fatigue failure probability can also be computed as a volumetric phenomenon, which can be expressed as

$$
p_{f, \text { volume }}=1-\exp \left(-\int_{V}\left(\frac{\sigma_{\text {eff }}-\sigma_{\mathrm{th}, \mathrm{vol}}}{\sigma_{\mathrm{u}, \mathrm{vol}}}\right)^{m} \frac{\mathrm{d} V}{V_{\text {ref }}}\right),
$$

where $V$ is the specimen volume and all other quantities have the same meaning as in Eq. (5). In order to compute the fatigue failure probability from FEresults, the integration in Eq. (6) turns is taken as a summation over the finite elements as

$$
p_{f, \text { volume }}=1-\exp \left(-\sum_{i=1}^{N_{\text {elem }}}\left(\frac{\sigma_{\text {eff }, i}-\sigma_{\text {th }, \mathrm{vol}}}{\sigma_{\mathrm{u}, \mathrm{vol}}}\right)^{m} \frac{V_{i}}{V_{\text {ref }}}\right) .
$$

In Eq. (7), $N_{\text {elem }}$ is the total number of finite elements in the model and all the other entities are the same as above. The same equation can be used for the area-method as well, in this case the summation is performed over the surface elements instead of the element volumes.

In order to compute the fatigue failure probability using the point stress, the normal distribution is used. The normal distribution is expressed as

$$
p_{f, \text { point }}=\Phi\left(\frac{\sigma_{\text {eff }}-\sigma_{\text {mean }}}{\sigma_{\text {std }}}\right) .
$$

where $\Phi$ denotes the cumulative distribution function of the normal distribution, $\sigma_{\text {eff }}$ is an effective stress, $\sigma_{\text {mean }}$ is the mean values and $\sigma_{\text {std }}$ is the standard deviation. The stress value in Eq. (8), $\sigma_{\text {eff }}$, can be either the largest occurring 
stress value, called the point stress $\left(\sigma_{\text {eff,point }}\right)$, or the gradient adjusted point stress

$$
\sigma_{\text {eff,grad }}=\sigma_{\text {eff,point }}-\gamma \cdot\left|\operatorname{grad}\left(\sigma_{\text {eff,point }}\right)\right| .
$$

In Eq. (9) $\gamma$ is a material parameter that controls the influence from the stress gradient. The gradient adjusted stress has proven to have good predictive capabilities, see $[1,10]$ for instance. Both the point stress value and the gradient adjusted point stress will be used.

In order to find the distribution parameters the following residual is minimized

$$
R e s^{2}=\sum_{i=1}^{n}\left(p_{f \text { model }, i}-p_{f \exp , i}\right)^{2}
$$

where $p_{f \text { model }, i}$ is the failure probability according to the fatigue limit model, Eq. (5), (6) or (8), $p_{f \text { exp }, i}$ is the median rank estimate, [20], of the fatigue failure probability from the experiments and $n$ is the number of data points used in the fit. The experimental failure probabilities are computed from the experimental outcome presented in Fig. 2 and Table 2.

Table 4: Parameters for the best fatigue limit models, minimizing Res in Eq. (10). Stresses are in MPa. The point stress is used when $\gamma=0$.

\begin{tabular}{ccccccc}
\hline$\gamma[\mathrm{mm}]$ & $\sigma_{\text {mean }}$ & $\sigma_{\text {std }}$ & $\sigma_{\text {th }}$ & $\sigma_{\mathrm{u}}$ & $m[-]$ & $\operatorname{Res}[-]$ \\
\hline \hline 0.44 & 205 & 9.94 & - & - & - & 0.156 \\
0 & 441 & 55.9 & - & - & - & 0.651 \\
- & - & - & 302 & 6.44 & 2.72 & 0.491 \\
- & - & - & 367 & 0.127 & 0.868 & 0.465 \\
\hline
\end{tabular}

In Table 4 the parameters from fitting the different models to the experimental data are presented. It can be seen that the best fit is obtained using the gradient adjusted point stress and the worst fit is from using the point stress. A comparison between the failure probability from the different fatigue limit models and the failure probability from the experiments is shown in Fig. 9.

The gradient adjusted point stress shows a very good agreement with the experimental outcome in Fig. 9. The parameters of all models are used for Fig. 10 , where the failure probability according to the different models is compared to the experimental failure probability for the different load cases used in the competing notch tests. The symbols in this figure are obtained from the load cases presented in Table 3. The experimental failure probability is estimated using median ranks, [20].

In Fig. 10 it can be seen that none of the four fatigue limit models can describe the experimental outcome. All four models show similar behavior, which does not correspond to the experimental behavior. 

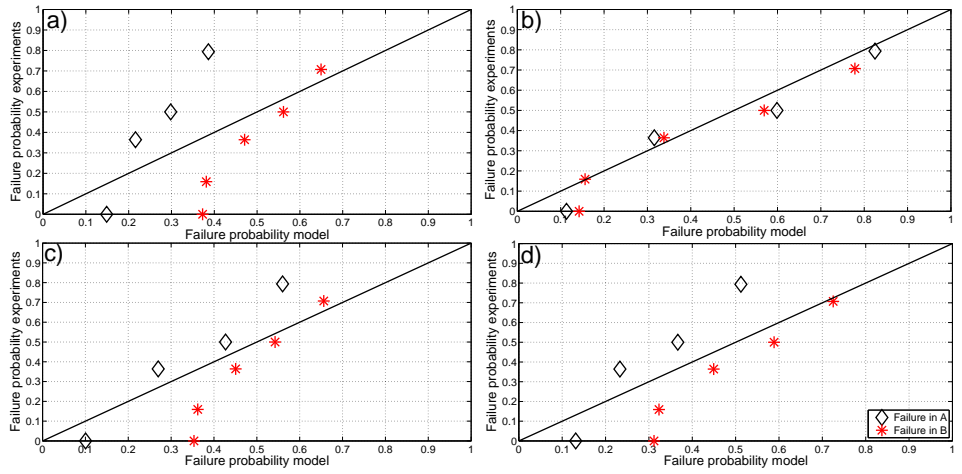

Figure 9: Comparison between experimental failure probability and failure probability for the different fatigue limit models where the diagonal line indicates a perfect fit. a) point stress, b) gradient adjusted point stress, c) area model and d) volume model.
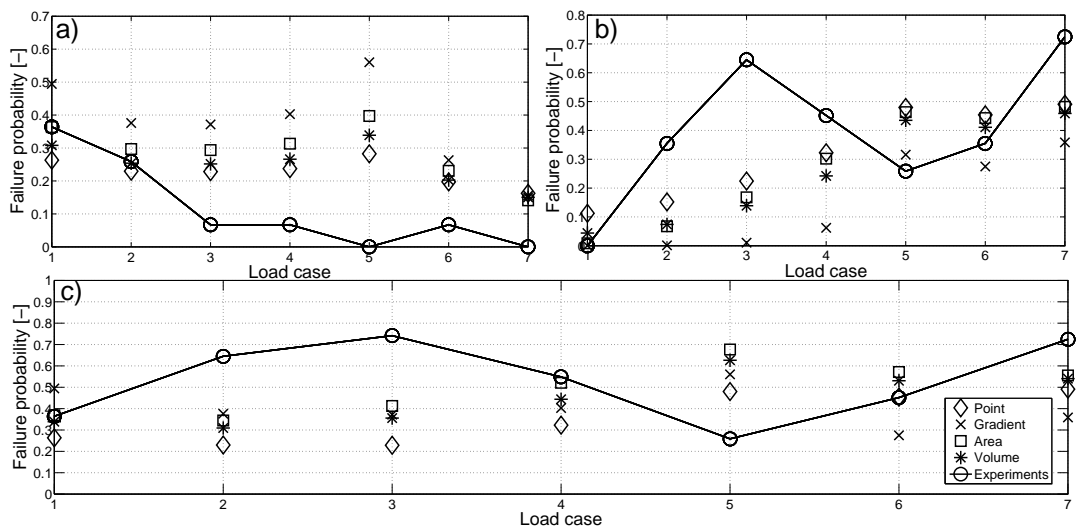

Figure 10: Experimental failure probabilities and predictions from the fatigue limit models for the CN-tests a) failure in just notch $A, \mathrm{~b}$ ) failure in just notch $B$ and c) the total failure probability. Load cases shown in Table 3.

\section{Analysis of the estimated fatigue failure loca- tions}

In order to understand the discrepancy between the experimental outcome and the model predictions, the stress was evaluated at the estimated fatigue failure locations. The point stress distributions around the notch tips from the fatigue limit tests are plotted in Fig. 11.

In Fig. 11 it can be seen that the failure location is different from one experiment to the other and never at the location where the stress is the largest, i.e. the diamonds. If the point stress is modified using the stress gradient, the 

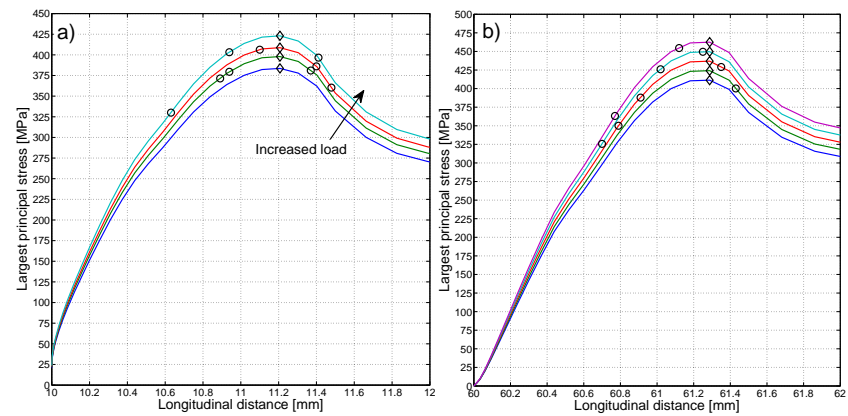

Figure 11: Point stress distribution around the notches as function of the longitudinal distance, the lines indicate different load levels. a) is notch $A$ and b) is notch $B$. The rings indicate estimated fatigue failure locations and the diamonds indicate the location of the largest stress.

difference in failure locations could be better understood since the difference in local stress would be smaller. Two ways to do this will be investigated here; 1) choosing $\gamma$ so that the local stress values are as equal as possible in the region containing all failure locations or 2) choosing $\gamma$ so that a smooth stress distribution in an area that is $90 \%$ of the maximal stress value (no failure locations are taken into account).

For the first method, the gradient parameter $\gamma$ is chosen so that the variation in the stress distribution at the notches is as small as possible. This is done by evaluating the stress at the estimated fatigue failure locations and then computing the combined coefficient of variation of the stress at that notch as

$$
C S=\sqrt{\left(\frac{\sigma_{\mathrm{std}, A}}{\sigma_{\text {mean }, A}}\right)^{2}+\left(\frac{\sigma_{\mathrm{std}, B}}{\sigma_{\text {mean }, B}}\right)^{2}} .
$$

In Eq. (11) $\sigma_{\text {mean }, A}$ and $\sigma_{\mathrm{std}, A}$ are the mean value and standard deviation of the stresses evaluated at notch $A$ and $\sigma_{\text {mean, } B}$ and $\sigma_{\text {std }, B}$ are the same quantities for notch $B$. The value of $\gamma$ is chosen so that $C S$ attains its smallest value.

Different stress gradient distributions for notches $A$ and $B$ are shown in Fig. 12. Three different values on $\gamma$ are used; $\gamma_{1}=0.440 \mathrm{~mm}$ is for having an optimal fit (i.e. lowest Res), $\gamma_{2}=0.184 \mathrm{~mm}$ is for minimizing $C S$ in Eq. (11) and $\gamma_{3}=0.268 \mathrm{~mm}$ is for a stress distribution as smooth as possible based on $0.9 \sigma_{\max }$.

Choosing a $\gamma$ that gives the smallest residual gives a rather smooth stress distribution, except for some failure locations, see Fig. 12a) and d). For the two other values on $\gamma$ it can be seen that the stress distributions are rather smooth. In order to compare these different stress distributions, the coefficient of variation ( $\operatorname{cov}=\mathrm{std} /$ mean) is computed for each notch for all the fatigue failure locations for all values on $\gamma$. The result from this is shown in Table 5 .

In Table 5 it can be seen that the cov is reduced when using the gradient adjusted point stress. The three gradient adjusted point stress distributions 

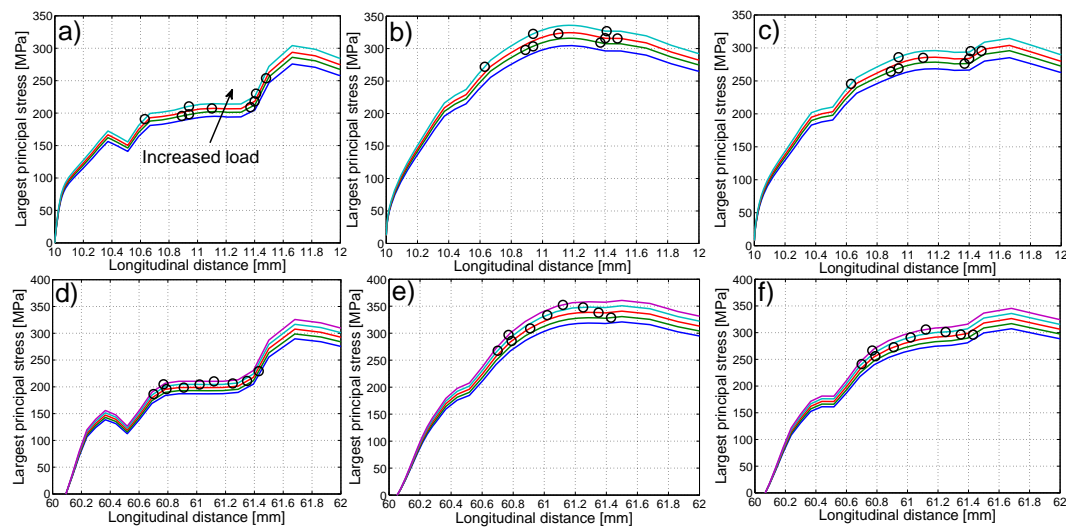

Figure 12: Stress gradient distributions around the notches for different values on the gradient parameter, $\gamma$. a) is best fit for $A\left(\gamma_{1}\right)$, b) is lowest $C S$ for $A$ $\left.\left(\gamma_{2}\right), \mathrm{c}\right)$ is for $0.9 \sigma_{\max }$ for $\left.\left.A\left(\gamma_{3}\right), \mathrm{d}\right), \mathrm{e}\right)$ and $\mathrm{f}$ ) are the same but for notch $B$.

Table 5: Stress gradient values where $\gamma=0$ means that the point stress is used. Stresses are in MPa.

\begin{tabular}{lcccccccccc}
\hline & \multicolumn{9}{c}{ Notch $A$} \\
$\gamma[\mathrm{mm}]$ & $\max$ & $\min$ & $\operatorname{mean}$ & std & cov $[\%]$ & $\max$ & $\min$ & mean & std & cov [\%] \\
\hline \hline 0 & 406 & 330 & 379 & 23.7 & 6.25 & 455 & 326 & 398 & 45.3 & 11.4 \\
$0.440\left(\gamma_{1}\right)$ & 254 & 191 & 212 & 19.5 & 9.16 & 229 & 187 & 205 & 11.9 & 5.77 \\
$0.184\left(\gamma_{2}\right)$ & 327 & 272 & 310 & 17.0 & 5.50 & 352 & 268 & 318 & 29.5 & 9.27 \\
$0.268\left(\gamma_{3}\right)$ & 295 & 245 & 278 & 16.1 & 5.80 & 306 & 241 & 281 & 22.6 & 8.05 \\
\hline
\end{tabular}

give similar values on the cov's.

The experimental and model failure probabilities for different stress gradient distributions are compared in Fig. 13.

In Fig. 13 it can be seen that if the stress is evaluated at the estimated fatigue failure location, the fit is poor. For the point stress the failure probability is in general underestimated, which is not the case for the gradient adjusted point stress.

In order to estimate the likeliness of getting the experimental outcome that is obtained, the local fatigue failure probability around the notches can be estimated. The failure probability is computed using the normal distribution for the point stress and the gradient adjusted point stress. In Fig. 14 the failure probability distributions for the point stress for the different load cases for notches $A$ and $B$ are shown.

In Fig. 14 it can be seen that some specimens fail in the notch with the lower failure probability. Further, the local failure probability is sometimes much lower than the maximal one. The curves presented are interpolations of the point-wise fatigue failure probabilities computed from the nodal stress 

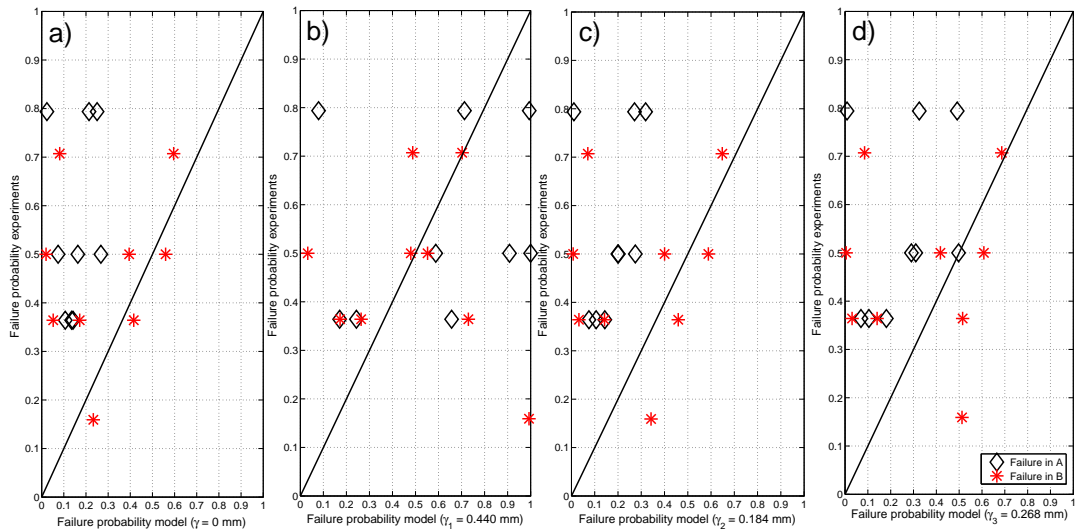

Figure 13: Comparison between the failure probability according to the different gradient adjusted stresses and experimental failure probability, when the stress is evaluated at the estimated fatigue failure location. a) is point criterion, b) gradient criterion with the best fit, c) gradient with lowest $C S$ and d) smoothest stress distribution based on $0.9 \sigma_{\max }$.
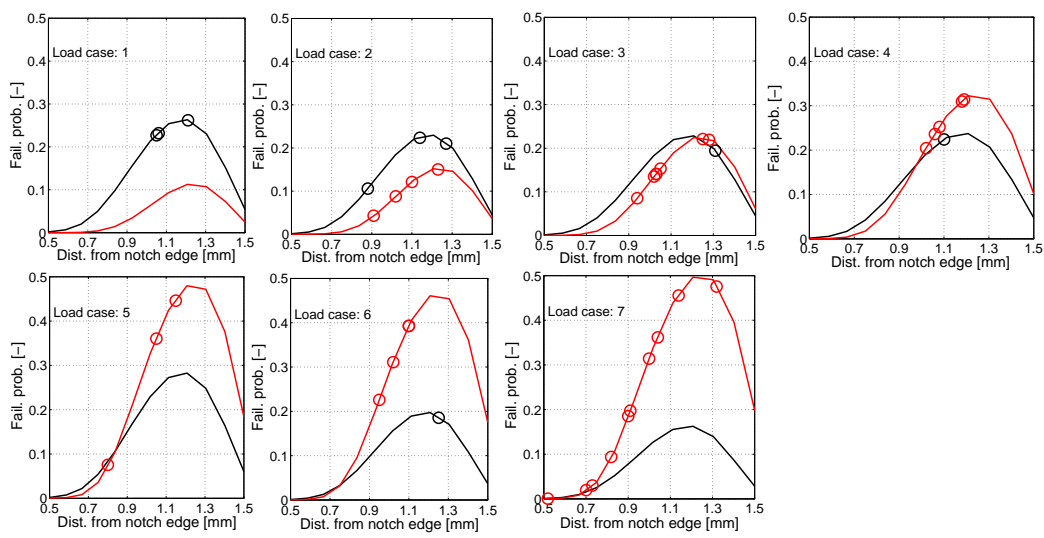

Figure 14: Failure probability distributions around the notches using the point method, black is notch $A$ and red is notch $B$. The circles indicate failure locations.

values. The failure probability distributions using the gradient adjusted point stress distribution with $\gamma=0.184 \mathrm{~mm}$ are shown in Fig. 15 .

Here, the gradient parameter is chosen so that $C S$ in Eq. (11) is minimized (i.e. $\gamma_{2}=0.184 \mathrm{~mm}$ ). Fig. 15 shows that the gradient adjusted stress can not explain the experimental outcome, i.e. fatigue failure location.

The fatigue failure probability can be computed using the WL-integral and performing the integration over the specimen surface area or over the volume. The functions in Fig. 16 and 17 show the element-wise contributions to the 

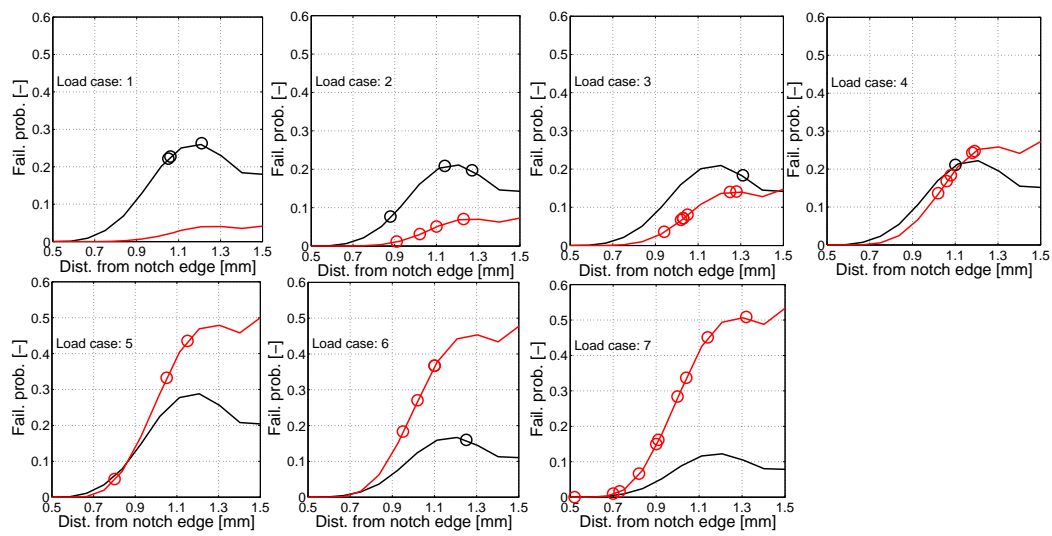

Figure 15: Failure probability distributions around the notches, gradient method with $\gamma_{2}$. The circles indicate failure locations.

failure probabilities in the notches for area and volume, respectively.
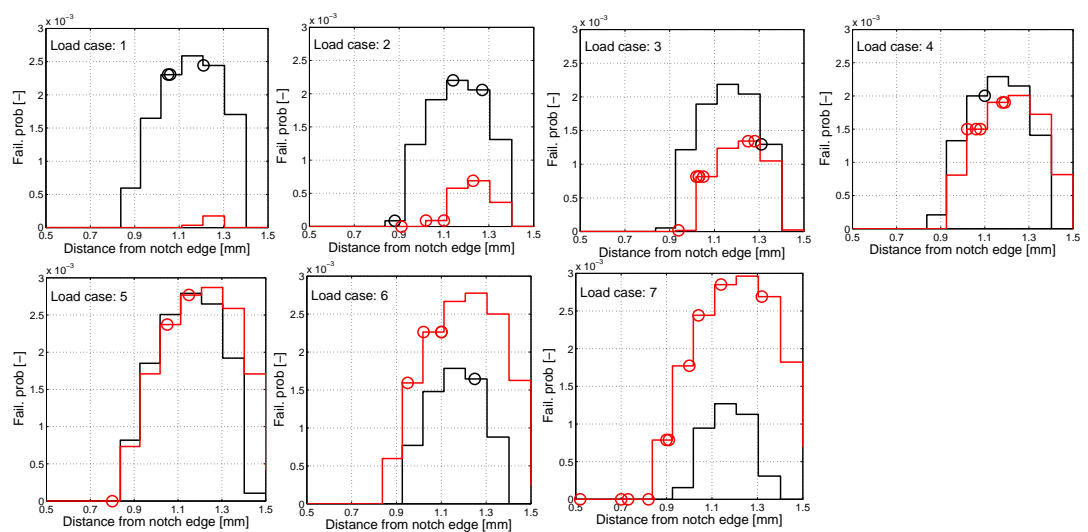

Figure 16: Contribution to failure probability from elements in the notches, area method. The function indicate the individual failure probability for one element in the notch root region. The circles indicate failure locations.

The element-wise failure probability for the area method is shown in Fig. 16. The trend is similar to the trend in Fig. 14 and 15. In Fig. 17 the element-wise failure probability according to the WL-integral using the volume method is shown.

In Fig. 17 the trend is similar as in Fig. 16. 

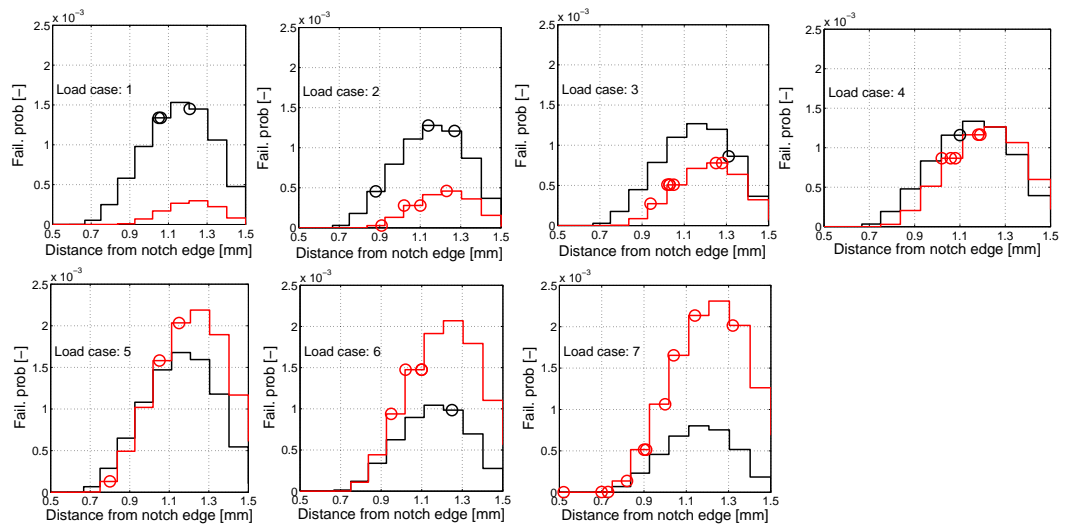

Figure 17: Failure probability in elements in the notches, volume method. The functions indicate the individual failure probability for one element in the notch root region. The circles indicate failure locations.

\section{Fatigue lives of the failed specimens}

In order to better understand the experimental outcome from the competing notch tests, the fatigue lives of the failed specimens were studied. The failure criterion for all specimens is complete fatigue failure. The fatigue lives for the failed specimens are shown in Fig. 18.

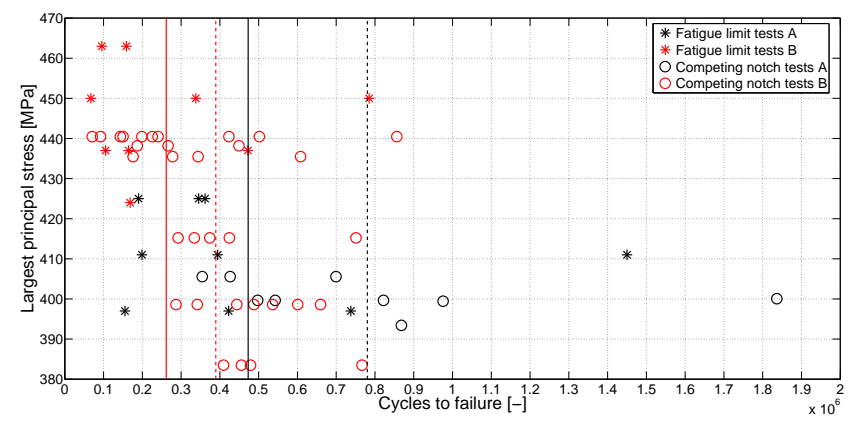

Figure 18: Fatigue lives for the experiments that failed. The filled lines indicate the mean values for the fatigue limit tests and the dashed lines for the competing notch tests.

In Fig. 18 the largest point stress is used and it is not evaluated at the estimated fatigue failure locations, hence it is the same for all failures per load case. It can be seen that the specimens that failed in notch $A$ generally have longer fatigue lives compared to notch $B$, which could have an influence on the outcome of the competing notch tests. 


\section{Discussion}

The estimated failure location were measured using a dial indicator, with a resolution of $\pm 0.01 \mathrm{~mm}$. All the measurements were performed in the same way, thus reducing the risk of an estimation error. In Fig. 6 and 7 it can be seen that there is large scatter present in the estimated fatigue failure locations. Even though there is a span for the edge measurements the estimated failure location is still different from one specimen to the other. All spans do not overlap each other, and thus there is substantial scatter present in the estimated locations that can not be neglected.

The experimental outcome from the competing notch tests is shown in Table 3. It can be seen that for $\beta=78 \mathrm{~mm}$ all specimen fail in $A$ and for $\beta=79 \mathrm{~mm}$ three fail in $A$ and four in $B$. In [1] most of the experiments fail in notch $A$ when the load is applied in the region $\beta=77.5 \mathrm{~mm} \beta=78.75 \mathrm{~mm}$. This is the same region as for the failure location switch for the competing notch tests. It should be noted that the experiments in [1] were all finite life tests whereas the experiments performed in this paper are fatigue limit tests.

In Fig. 10 the model prediction from the competing notch tests is shown. It can be seen that none of the four models can capture the trend in the experiments.Not even the gradient adjusted point stress, which showed good predictive capabilities in Fig. 9, can capture the experimental trend. The reason for this discrepancy needs to be investigated further.

In order to better understand the experimental outcome from the experiments with competing notch tests, the stress gradient parameter was chosen so that the stress distribution was as smooth as possible over the failure location range. In Table 5 it can be seen that the relative difference in local stress is reduced when using different stress gradient values compared to using the point stress. In Fig. 12 it can be seen that the stress distribution for $\gamma_{1}$ is rather smooth for most of the failures. When looking at the stress distribution for $\gamma_{2}$ the maximal stress is found in the region where all specimens fail. For $\gamma_{3}$, the stress distribution is not much higher for the two most extreme failures (in $A$ ) but is not as smooth for all failures. When choosing $\gamma_{3}$, the range where the stress is $90 \%$ of its maximal is supposed to be as smooth as possible, the lowest cov is obtained. It can be concluded that if $\gamma_{1}$ is used, the lowest Res is found. The residual is increased a bit if $\gamma_{2}$ is used, i.e. minimized $C S$, but the maximal stress is present in the range where all specimens fail. This is a realistic outcome, that the maximal stress is found where the specimens fail. When using $\gamma_{3}$, smoothest stress distribution based on $0.9 \sigma_{\max }$, the stress distributions have the lowest cov. However, the maximal stress is found outside the failure region, which is unrealistic.

When investigating the fatigue failure distributions around the notches it can be seen that some of the experimental outcomes are very unlikely. According to Fig. 14 the failure probability distributions are almost the same for load case 3 for the point stress. For this load case, seven specimens failed in notch $B$ and one in $B$, which is contradictory to the experimental outcome. For the gradient adjusted stress, Fig. 15, equally many experiments would fail in notch $A$ and 
$B$ for load case 4 , where in reality only one specimen failed in notch $A$ and four in notch $B$. For the area and volume stress, Fig. 16 and 17, equally many specimens should fail in both notches for load cases 4 and 5 , respectively. This is not the experimental outcome. This gives further evidence that the models presented in this paper can not describe the experimental outcome when there are two competing fatigue failure sites, i.e. notches. For $\gamma_{1}$ and $\gamma_{3}$ equally many specimens would fail in both notches for load cases 6 and 4 , respectively. If the fatigue limit parameters from the staircase test results are used along with the point stress, the outcome is even more unrealistic.

The reason for the experimental outcome in the competing notch tests might be defects. If random defects are considered, they could have a large influence on the fatigue properties of the two notches. The crack growth life for notch $B$ is shorter than for $A$, since notch $B$ has a smaller diameter. This could influence the competing notch tests. If two notches are competing towards failure and a crack has to grow a shorter distance, i.e. short life, in one of the notches, then fatigue failure should be more frequent in that notch.

Another factor that could explain the outcome in the competing notch tests is microplasticity. If such plasticity occurs, then the average stress state will be altered, thus giving rise to the difference in the fatigue failure location.

The fatigue lives for the specimens that failed in notch $A$ are generally longer than for $B$, see Fig. 18. This holds for both the fatigue limit tests and the competing notch tests. For the finite life tests, the fatigue lives are similar for notches $A$ and $B$. If the fatigue failure probability is the same for both notches but the expected fatigue life is different, then it can be assumed that the specimens will fail in the notch where the fatigue life is the shortest.

\section{Summary and Conclusions}

The following conclusions can be drawn:

- There is substantial scatter in the estimated fatigue failure locations, both for the fatigue limit tests and the competing notch tests.

- The different fatigue limit models can not describe the outcome from the competing notch tests.

- The gradient adjusted stress is is relatively smooth in the region of the fatigue failure locations.

- The experimental load case where equally many specimens fail in both notches is not the same as the one predicted by the four fatigue limit models.

- The fatigue lives are generally longer for notch $A$ compared to $B$. 


\section{Acknowledgments}

The authors would like to thank Scania CV AB for the financial support that made this investigation possible. M.Sc. Martin Öberg is thanked for help in design of the specimens and planning of the experiments. Mr. Kurt Lindquist is acknowledged for manufacturing all specimen.

\section{References}

[1] K. Karlén, M. Olsson, A probabilistic model for the entire HCF domain based on equivalent stress - Simulations and experiments, International Journal of Fatigue 36 (1) (2012) 9-17.

[2] T. Sakai, T. Sakai, K. Okada, M. Furuichi, I. Nishikawa, A. Sugeta, Statistical fatigue properties of SCM435 steel in ultra-long-life regime based on JSME database on fatigue strength of materials, International Journal Of Fatigue 28 (11) (2006) 1486-1492.

[3] K. Karlén, M. Olsson, A study of the volume effect and scatter at the fatigue limit - experiments and computations for a new specimen with separated notches, International Journal of Fatigue 33 (3) (2011) 363-371.

[4] K. Karlén, M. Olsson, An investigation of a fatigue model with two competing failure mechanisms, Submitted for for publication in International Journal of Fatigue (2012).

[5] J. Böhm, Zur vorhersage von dauerschwingfestigkeiten ungekerbter und gekerbter bauteile unter berücksichtigung des statistischen grössinflusses, Ph.D. thesis, Technische Universität München (1980).

[6] W. Magin, Untersuchnung des geometrischen grösseneinflusses bei umlauufbiegebeanspruchund unter besonderer berücksichtigung techonologischer einflüsse, Ph.D. thesis, Technische Hochschule Darmstadt (1981).

[7] W. Weibull, A statistical theory of the strength of materials, Tech. Rep. 151, Ingeniørsvetenskapsakademins handlingar (1939).

[8] W. Weibull, The phenomenon of rupture in solids, Tech. Rep. 153, Ingeniørsvetenskapsakademins handlingar (1939).

[9] K. Karlén, M. Olsson, Experimental and statistical investigation of the weakest link integral and the volume effect, Procedia Engineering 2 (1) (2010) 1451-1457.

[10] S. Norberg, M. Olsson, The effect of loaded volume and stress gradient on the fatigue limit, International Journal of Fatigue 29 (12) (2007) 2259-2272.

[11] F. Morel, L. Flacelière, Data scatter in multiaxial fatigue: from the infinite to the finite life regime, International Journal of Fatigue 27 (9) (2005) 1089-1101. 
[12] D. B. Lanning, T. Nicholas, A. Palazzo, HCF notch predictions based on weakest-link failure models, International Journal of Fatigue 25 (9-11) (2003) 835-841.

[13] I. Chantier, V. Bobet, R. Billardon, F. Hild, A probabilistic approach to predict the very high cycle fatigue behaviour of spheroidal cast iron structures, Fatigue \& Fracture of Engineering Materials and Structures 23 (2) (2000) 173-180.

[14] T. Delahay, T. Palin-Luc, Estimation of the fatigue strength distribution in high-cycle multiaxial fatigue taking into account the stress-strain gradient effect, International Journal of Fatigue 28 (5-6) (2006) 474-484.

[15] R. E. Peterson, Stress concentration factors, 1st Edition, John Wiley \& Sons, 1974.

[16] ASTM, STP 91-A: A Guide for Fatigue Testing and Analysis of Fatigue Data, 2nd Edition, ASTM, 1963.

[17] ASM International Handbook Committee, ASM Handbook 12 Fractography, Ninth Edition, ASM International, 1992.

[18] Hibbit, Karlsson, Sørensen, Abaqus/standard User's manual version 6.7.1 (2007).

[19] S. Norberg, M. Olsson, A fast, versatile fatigue post-processor and criteria evaluation, International Journal of Fatigue 27 (10-12) (2005) 1335-1341.

[20] L. Råde, B. Westergren, Mathematics Handbook for Science and Engineering, 4th Edition, Studentlitteratur, 2001. 